\title{
Removal from adherent culture contributes to apoptosis in human bone marrow mesenchymal stem cells
}

\author{
BAOPING DENG ${ }^{1,2}$, HAIMING JIANG ${ }^{2}$, KUAN ZENG $^{1}$, YI LIANG ${ }^{2}$, YINMENG WU $^{2}$ and YANQI YANG \\ ${ }^{1}$ Department of Cardiovascular Surgery, Sun Yat-sen Memorial Hospital, Sun Yat-sen University, \\ Guangzhou, Guangdong 510120; ${ }^{2}$ Department of Cardiothoracic Surgery, Zhongshan \\ People's Hospital, Zhongshan, Guangdong 528403, P.R. China
}

Received December 21, 2015; Accepted January 27, 2017

DOI: $10.3892 / \mathrm{mmr} .2017 .6440$

\begin{abstract}
Mesenchymal stem cells (MSCs) are routinely isolated due to their adherence to tissue culture plates and their in vitro growth characteristics. Expansion of MSCs in adherent cultures is the only way to obtain sufficient cells for use in either clinical or research settings. MSCs have tremendous potential in myocardial repair treatment by cell therapy techniques, however, a large number of MSCs die from apoptosis following transplantation. Previous studies have examined the factors contributing to the survival of transplanted cells, but little is known about the effect of removal from adherent culture conditions on apoptosis of the MSCs. In the present study, human bone marrow MSCs were expanded in adherent cultures. Then apoptosis rates were examined at different time points in MSCs cultured in nonadherent conditions (ultra-low-adherence plates) compared with MSCs cultured in adherent conditions (standard tissue culture plates). Flow cytometry analysis suggested that cell apoptosis increased when MSCs were cultured in nonadherent culture conditions. In addition, western blot and reverse transcription-quantitative polymerase chain reaction analyses demonstrated that caspase- $3,-7$ and -9 were involved in this process. The present study demonstrated that loss of culture adherence increases apoptosis of human MSCs. The present findings may provide new insight into the factors affecting MSC survival after transplantation.
\end{abstract}

\section{Introduction}

Mesenchymal stem cells (MSCs) are a promising cell source for regenerative medicine therapies, primarily due to their

Correspondence to: Professor Yanqi Yang, Department of Cardiovascular Surgery, Sun Yat-sen Memorial Hospital, Sun Yat-sen University, 74 Yanjiangxi Road, Guangzhou, Guangdong 510120, P.R. China

E-mail: yanqiyang_lio@yahoo.se

Key words: nonadherent culture, adherent culture, human mesenchymal stem cells, apoptosis multipotency and immunosuppressive functions (1). MSCs are also used for cell therapy in myocardial repair (2) and to reduce scar size following acute myocardial infarction (3). However, the therapeutic use of MSCs has been limited by many factors, including the difficulty of obtaining sufficient numbers of cells. Current methods include the in vitro expansion of MSCs in plastic adherent culture prior to in vivo transplantation (4), but the benefits of this cell transfer method are modest and short-lived. This may be partially attributed to poor survival and retention of the transplanted cells (5), which limits successful cell therapy for cardiac repair. Previous studies have identified multiple factors that are responsible for the poor survival of MSCs following transplantation into the myocardium, including ischemia, inflammatory response, hypoxia, and oxidative stress $(6,7)$.

Vunjak-Novakovic and Scadden (8) have classified the cellular and acellular components of the stem cell niche. The plastic adherent condition is considered a suitable niche for amplification of MSCs in vitro, promoting cells survival. To determine the effect of culture conditions on apoptosis of human MSCs (hMSCs), hMSCs were cultured in vitro either in ultra-low-adherence culture plates (to mimic nonadherent conditions) or in standard tissue culture plates (to mimic adherent conditions), as previously described (9). Apoptosis was then analyzed by flow cytometry. In addition, mRNA and protein expression levels of caspase-3, -7, -8, and -9 were analyzed by reverse transcription-quantitative polymerase chain reaction (RT-qPCR) and western blot analysis respectively. The present study demonstrated that removal from adherent culture conditions resulted in hMSC apoptosis through activation of the caspase pathway.

\section{Materials and methods}

Isolation and expansion of hMSCs. Bone marrow was aspirated from the posterior iliac crest of three healthy adult volunteers. All procedures were performed with the approval of the ethics committee of SunYat-sen Memorial Hospital, Sun Yat-sen University (approval number no. 2014-57; Guangzhou, China) and following informed written consent by the volunteers. Nucleated cells were isolated with a density gradient (Lymphoprep, Stemcell Technologies, Inc., Vancouver, Canada) and resuspended in MSC culture medium (MesenCult 
Proliferation kit; Stemcell Technologies, Inc.), according to the manufacturer's instructions. Nucleated cells $\left(1.2 \times 10^{7}\right)$ were plated in $20 \mathrm{ml}$ culture medium in T75 tissue culture flasks (Corning, Inc., Corning, NY, USA) and incubated at $37^{\circ} \mathrm{C}$ with $5 \% \mathrm{CO}_{2}$ and $20 \% \mathrm{O}_{2}$. Following $24 \mathrm{~h}$, nonadherent cells were discarded and adherent cells were thoroughly washed twice with PBS. The culture medium was changed every three days and following 10 days in culture, cells were $80 \%$ confluent. The cells were then incubated with $0.05 \%$ trypsin-EDTA (Gibco; Thermo Fisher Scientific, Inc., Waltham, MA, USA) for $5 \mathrm{~min}$ at $37^{\circ} \mathrm{C}$, and replated at 2,500 cells $/ \mathrm{cm}^{2}$ in T75 tissue culture flasks. Following 5 days incubation, the cultured cells were $80 \%$ confluent and were suspended by incubation in $0.05 \%$ trypsin-EDTA for $5 \mathrm{~min}$ at $37^{\circ} \mathrm{C}$ and rinsed with $5-7 \mathrm{ml}$ culture medium, followed by collection in a $50-\mathrm{ml}$ centrifuge tube. Cells were subsequently centrifuged at $300 \times \mathrm{g}$ (Sorvall $^{\mathrm{TM}}$ ST 16R; Thermo Fisher Scientific, Inc.) for $5 \mathrm{~min}$ at room temperature. Pellets were washed with culture medium and centrifuged at $300 \mathrm{x} \mathrm{g}$ for $5 \mathrm{~min}$ at room temperature again, following which the cells were resuspended in $10 \%$ dimethyl sulfoxide and $90 \%$ fetal bovine serum (FBS; Gibco; Thermo Fisher Scientific, Inc.) at $2 \times 10^{5}$ cells per freezing vial, and frozen in liquid nitrogen (passage-1 cells). To expand a culture, a frozen vial of MSCs was thawed, plated in a T75 tissue culture flask, and incubated for 5 days at $37^{\circ} \mathrm{C}$ with $5 \%$ $\mathrm{CO}_{2}$ and $20 \% \mathrm{O}_{2}$ (passage-2 cells).

Fluorescence-Activated Cell Sorting (FACS) analysis of MSC surface markers. Passage-1 cells were plated in T75 tissue culture flasks after being thawed from liquid nitrogen, and incubated at $37^{\circ} \mathrm{C}$ with $5 \% \mathrm{CO}_{2}$ and $20 \% \mathrm{O}_{2}$. When the cultured cells reached $90 \%$ confluency (passage- 2 cells), they were harvested, centrifuged at $300 \mathrm{x} g$ for $5 \mathrm{~min}$, and resuspended in PBS. Cells were then stained with mouse anti-human CD11b(cat.no.557321; 1:100),CD14(cat.no.557154; 1:100),CD34 (cat. no. 550619; 1:100), CD45 (cat. no. 555482; 1:100), CD73 (cat. no. 561014; 1:100), CD90 (cat. no. 555596; 1:100) and CD105 (cat. no. 561443; 1:100) antibodies (BD Biosciences, Franklin Lakes, NJ, USA) and analyzed by flow cytometry using a BD FACSCalibur (BD Biosciences). An isotype control immunoglobulin (cat no. PE-R3-34; BD Biosciences) was used at the indicated concentration. BD FACSComp software (version no. 5.1) was used for the data analysis (BD Biosciences).

Colony-Forming Unit-Fibroblast (CFU-F) assay. Passage-2 cells were plated into 6 -well plates at 100 cells/well in complete Dulbecco's modified Eagle's medium (DMEM; Gibco; Thermo Fisher Scientific, Inc.) containing 10\% FBS, 10 mM N-2-hydroxyethylpiperazine-N-2-ethane sulfonic acid (HEPES; Gibco; Thermo Fisher Scientific, Inc.), $100 \mathrm{U} / \mathrm{ml}$ penicillin, $100 \mu \mathrm{g} / \mathrm{ml}$ streptomycin and incubated at $37^{\circ} \mathrm{C}$ with $5 \% \mathrm{CO}_{2}$ and $20 \% \mathrm{O}_{2}$ for 12 days. The colonies were stained with Giemsa (Sigma-Aldrich; Merck Millipore, Darmstadt, Germany). A cluster of $>50$ cells was counted as one colony, and images were acquired using a Leica Application Suite software (version no. 4.0; Leica Microsystems GmbH, Wetzlar, Germany).

In vitro differentiation assay. The assay was performed with passage- 2 cultured hMSCs according to a previously described protocol (10), for evaluation of their multipotent differentiation ability. The cells were split into three separate differentiation-specific media to induce osteoblast, adipocyte, or chondrocyte differentiation. The media were changed every 3-5 days for 3-4 weeks. For adipocyte differentiation, the cultures were incubated in DMEM containing $10 \%$ FBS, $10 \mu \mathrm{M}$ HEPES, $100 \mathrm{U} / \mathrm{ml}$ penicillin, $100 \mu \mathrm{g} / \mathrm{ml}$ streptomycin, $5 \mu \mathrm{g} / \mathrm{ml}$ insulin, $20 \mu \mathrm{M}$ indomethacin, $0.5 \mu \mathrm{M}$ isobutylmethylxanthine (Sigma-Aldrich; Merck Millipore), and $1 \times 10^{-7} \mathrm{M}$ dexamethasone (Sigma-Aldrich; Merck Millipore). Following 28 days' incubation, the cells were fixed with $4 \%$ formalin and stained with $0.5 \%$ oil red O. For osteoblast differentiation, the cells were cultured in DMEM containing 10\% FBS, $10 \mathrm{mM}$ HEPES, $100 \mathrm{U} / \mathrm{ml}$ penicillin, $100 \mathrm{U} / \mathrm{ml}$ streptomycin, $50 \mu \mathrm{g} / \mathrm{ml}$ ascorbic acid (Sigma-Aldrich; Merck Millipore), $1 \times 10^{-7} \mathrm{M}$ dexamethasone and $10 \mathrm{mM}$ glycerol phosphate. Following 21 days' incubation, the cells were fixed with $4 \%$ formalin and stained with $1 \%$ alizarin red $\mathrm{S}(\mathrm{pH} 4.1)$. Chondrocyte differentiation was induced with OriCell Human Mesenchymal Stem Cell Chondrogenic Differentiation Medium (Cyagen Biosciences, Guangzhou, China), according to the manufacturer's instructions. The chondrogenic pellets were harvested following 18 days in culture, fixed with $4 \%$ formalin, and embedded in paraffin for Alcian blue staining. Adipocyte and chondrocyte differentiation assays were carried out similarly for both adherent and nonadherent cultured cells at $72 \mathrm{~h}$ to evaluate their differentiation potential.

Expansion of MSCs and nonadherent in vitro culture conditions. Passage-2 cultures were plated in $20 \mathrm{ml}$ complete DMEM containing 10\% FBS, 10 mM HEPES, $100 \mathrm{U} / \mathrm{ml}$ penicillin, and $100 \mu \mathrm{g} / \mathrm{ml}$ streptomycin in T75 tissue culture flasks. The cells were incubated at $37^{\circ} \mathrm{C}$ with $20 \% \mathrm{O}_{2}$ and $5 \%$ $\mathrm{CO}_{2}$. The medium was changed every 5-7 days. When MSCs reached $90 \%$ confluency, they were suspended by incubation in $0.05 \%$ trypsin-EDTA for $5 \mathrm{~min}$ at $37^{\circ} \mathrm{C}$ and then reseeded at 2,500 cells $/ \mathrm{cm}^{2}$ in a T75 tissue culture flask (passage- 3 cells). To test the effect of adherent vs. nonadherent culture conditions in MSCs, passage 3 cells were seeded at 2,500 cells $/ \mathrm{cm}^{2}$ in adherent culture plates (BD Biosciences) or in ultra-low-adherence tissue culture plates (Corning, Inc.) respectively, and allowed to grow for 24 or $72 \mathrm{~h}$ prior to experimental assays.

Flow cytometry assay for apoptosis. Adherent-cultured cells and nonadherent-cultured cells grown for 24 or $72 \mathrm{~h}$ were collected in $1.5 \mathrm{ml}$ Eppendorf (EP) tubes and centrifuged at $300 \mathrm{x} g$ for $5 \mathrm{~min}$ at room temperature. Pellets were washed twice with PBS, followed by resuspension in $400 \mu \mathrm{l}$ binding buffer (as per the kit instructions; eBioscience, Inc., San Diego, CA, USA), and divided equally into two new tubes. One sample was used as the negative control, while $5 \mu \mathrm{l}$ Annexin V-FITC (eBioscience, Inc.) was added to the other sample and incubated at room temperature for $15 \mathrm{~min}$. Propidium iodide (10 $\mu \mathrm{l})$ was added to both samples, and they were analyzed by flow cytometry on a FACSCalibur using CellQuest software (version no. 3.3; BD Biosciences).

$R T-q P C R$. Adherent-cultured cells and nonadherent-cultured cells grown for 24 or $72 \mathrm{~h}$ were collected in 50-ml centrifuge tubes and centrifuged at $300 \mathrm{x} g$ for $5 \mathrm{~min}$ at room temperature. 


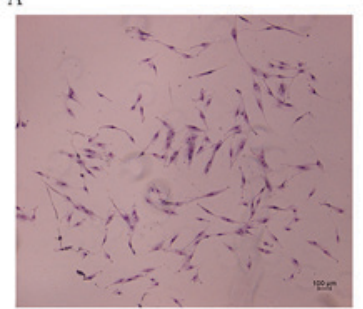

E

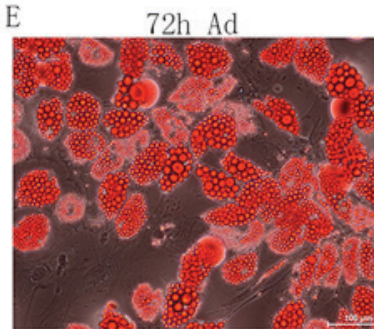

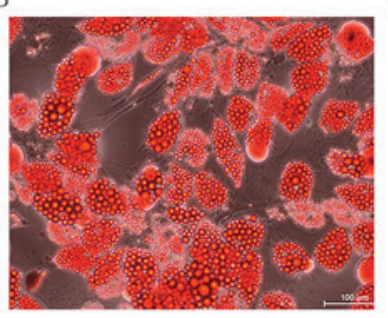

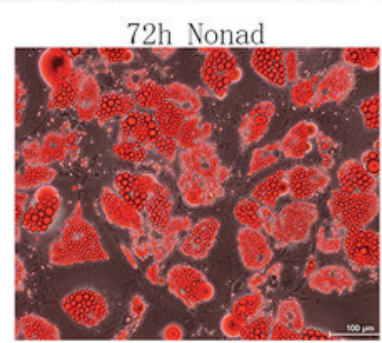

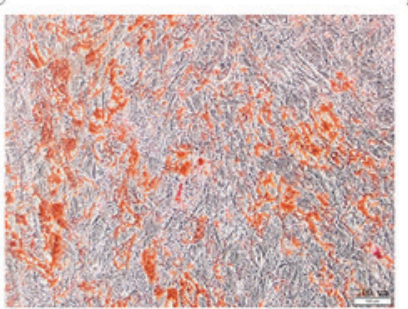

$72 \mathrm{~h} \mathrm{Ad}$

D

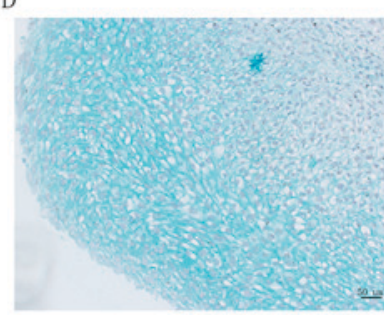

$72 \mathrm{~h}$ Nonad
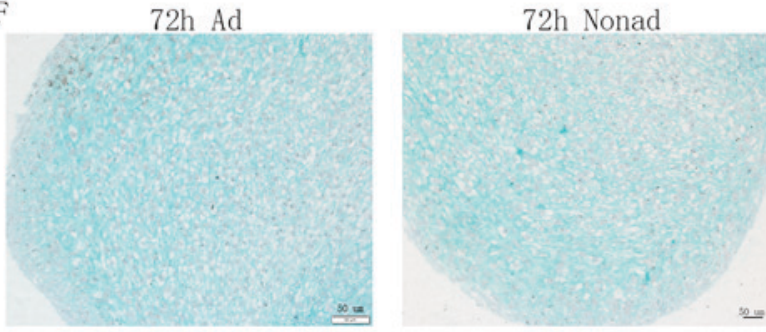

Figure 1. CFU-F and differentiation assays. (A) Morphology of Giemsa-stained CFUs, with a typical colony of passage-2 hMSCs depicted in the image. (B) In vitro adipocyte differentiation of passage- $2 \mathrm{hMSCs}$ was confirmed by oil red O staining. (C) In vitro osteoblast differentiation of passage-2 hMSCs was confirmed by alizarin red staining. (D) In vitro chondrocyte differentiation of passage-2 hMSCs was confirmed by Alcian blue staining. (E) Adherent or nonadherent-cultured cells at $72 \mathrm{~h}$ were reseeded in tissue culture plates for adipocyte differentiation and adipocytes were confirmed by oil red $\mathrm{O}$ staining. (F) Adherent or nonadherent-cultured cells at $72 \mathrm{~h}$ were reseeded in tissue culture plates for chondrocyte differentiation and chondrocytes were confirmed by Alcian blue staining. CFU, colony forming unit; F, fibroblast; hMSC, human mesenchymal stem cells; Ad, adherent culture; Nonad, nonadherent culture.

Pellets were resuspended with Dulbecco's PBS and transferred into $1.5 \mathrm{ml}$ EP tubes. Total RNA was extracted using the High Pure RNA Isolation kit (Roche Diagnostics, Ltd., Basel, Switzerland), according to the manufacturer's instructions. cDNA was synthesized from $1 \mu \mathrm{g}$ RNA using SuperScript III and Oligo primers (Thermo Fisher Scientific, Inc.), according to the manufacturer's instructions. Primers were as follows: caspase-3, forward 5'-CTGGACTGTGGCATTGAGAC-3' and reverse 5'-ACAAAGCGACTGGATGAACC-3'; caspase-7, forward 5'-CAAGATCCCAGTGGAAGCTGAC-3' and reverse 5'-GGCTTGCACAAACCAGGAG-3'; caspase-8, forward 5'-GAAGGTGCTACCATCGTGAGAGTAA-3' and reverse 5'-CCTGGAGTCTCTGGAATAACATCAA-3'; caspase-9, forward 5'-CGCAAACCAGAGGTTCTCAGAC-3' and reverse 5'-AGGATGTAAGCCTGCCAGCAC-3'; and GAPDH, forward 5'-GCACCGTCAAGGCTGAGAAC-3' and reverse 5'-TGG TGAAGACGCCAGTGGA-3'. qPCR analysis was performed using the LightCycler 480 platform and the LightCycler 480 SYBR-Green I Master kit (Roche Diagnostics, Ltd.). Each reaction mixture contained $10 \mathrm{ml}$ SYBR Green I master mix, $6 \mu \mathrm{l}$ RNase-free $\mathrm{H}_{2} \mathrm{O}, 1 \mu \mathrm{l} 10 \mathrm{mM}$ forward primer, $1 \mu 110 \mathrm{mM}$ reverse primer, and $2 \mu \mathrm{l}$ cDNA (undiluted) in a final reaction volume of $20 \mathrm{ml}$. Gene expression was normalized to the internal reference gene $\beta$-actin. The $2^{-\Delta \Delta C q}$ method (11) was used to determine relative fold changes using the LightCycler480 software (Roche Diagnostics, Ltd.). The experiment was performed three independent times in triplicate.

Western blot analysis. Adherent-cultured cells and nonadherent-cultured cells grown for 24 or $72 \mathrm{~h}$ were lysed on ice for 5 min using Cell Lysis Buffer (Cell Signaling Technology, Inc., Danvers, MA, USA) containing $1 \mathrm{mM}$ phenylmethanesulfonyl fluoride (Beyotime Institute of Biotechnology, Haimen, China) and protease inhibitors (Roche Diagnostics, Ltd.), then sonicated briefly and centrifuged for $10 \mathrm{~min}$ at 14,000 $\mathrm{x} g$ in a cold microfuge, according to the manufacturer' protocol. Protein concentrations were determined using the bicinchoninic acid method (Beyotime Institute of Biotechnology). Total protein samples (30 $\mu$ g per lane) were separated by $8 \%$ SDS-PAGE and transferred to polyvinylidene fluoride membranes (Beyotime Institute of Biotechnology). Following blocking with 5\% dried skim milk in TBS/0.1\% Tween-20 (TBST) for $1 \mathrm{~h}$ at room temperature, the membranes were incubated overnight at $4 \beta$ with primary antibodies (1:1,000 dilution) against caspase-3 (cat. no. 9665; Cell Signaling Technology, Inc.), caspase-7 (cat. no. 12,827; Cell Signaling Technology, Inc.), caspase-8 (cat. no. AC056; Beyotime Institute of Biotechnology), caspase-9 (cat. no. 9508; Cell Signaling Technology, Inc.) and $\beta$-actin (cat. no. AA128; Beyotime Institute of Biotechnology) in primary antibody dilution buffer. Following 3 washes with TBST, membranes were incubated with horseradish peroxidase (HRP)-linked anti-mouse immunoglobulin (Ig) G antibody (cat. no. 7076; 1:2,000 dilution; Cell Signaling Technology, Inc.) or HRP-linked anti-rabbit IgG antibody (cat. no. 7074; 1:2,000 dilution; Cell Signaling Technology, Inc.) for $1 \mathrm{~h}$ at room temperature. Protein expression signals were detected with BeyoECL Plus enhanced chemiluminescence reagent (Beyotime Institute of Biotechnology) using the Intelligent imaging system (Syngene, Frederick, MD, USA) and ImageJ software (version no. 1.44) (12), according to the manufacturer's instructions.

Statistical analysis. GraphPad Prism 5 (GraphPad Software, Inc., La Jolla, CA, USA) was used to perform Student's $t$-test analysis in order to identify significant differences between culture conditions. $\mathrm{P}<0.05$ was considered to indicate a statistically significant difference.

\section{Results}

$C F U-F$ and in vitro differentiation assays. Growth and multipotency are key features of MSCs. To confirm the functional 
A
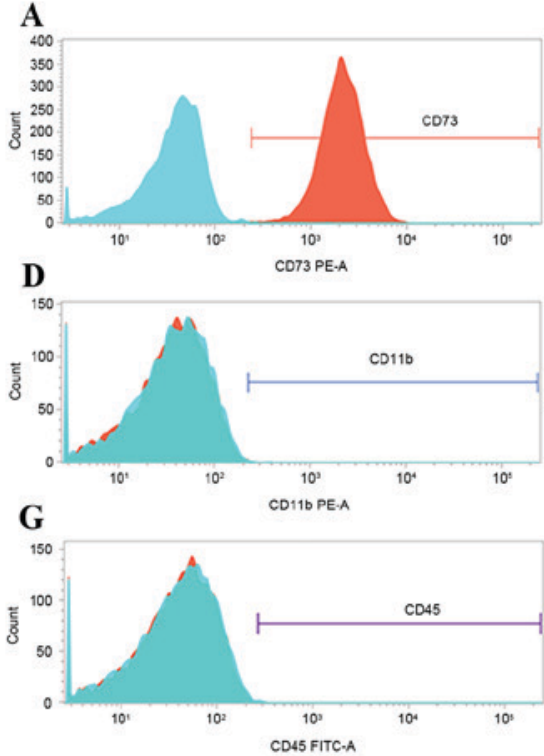

\section{B}

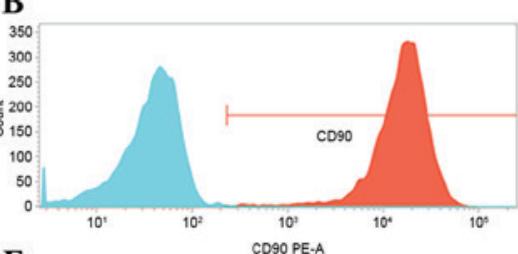

$\mathbf{E}$

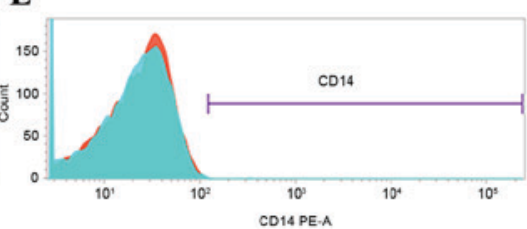

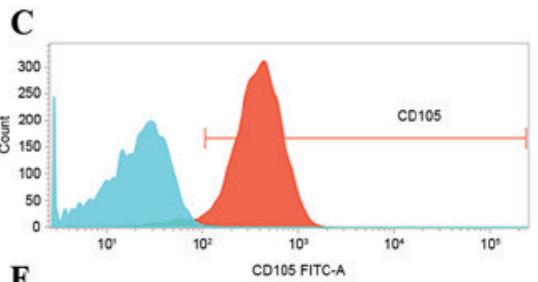

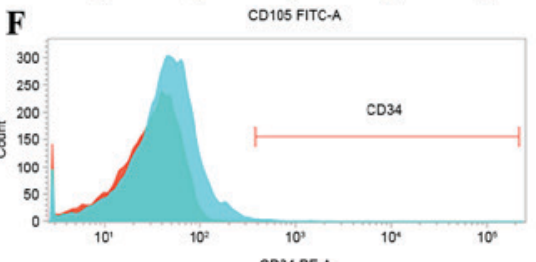

Figure 2. Flow cytometric analysis of MSC surface markers. Representative flow cytometry profiles are depicted here for (A) CD73, (B) CD90, (C) CD105, (D) CD11b, (E) CD14, (F) CD34 and (G) CD45. Blue plots represent the negative control, IgG-stained cells, while red plots represent the surface marker antibody-stained cells. PE, phycoerythrin; FITC, fluorescein isothiocyanate.

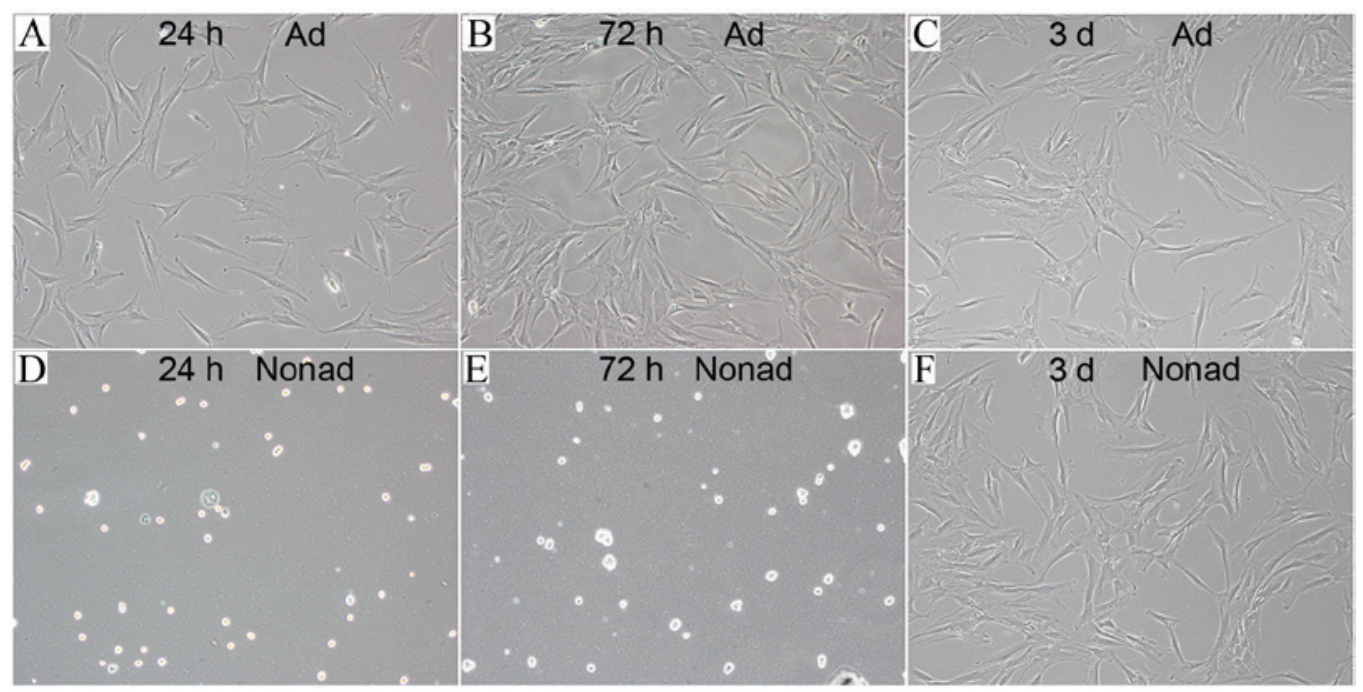

Figure 3. Morphologies of hMSCs in adherent and nonadherent cultures. (A) hMSCs plated in tissue culture plate for $24 \mathrm{~h}$. (B) hMSCs plated in tissue culture plate for $72 \mathrm{~h}$. (C) Adherent culture cells from panel B at $72 \mathrm{~h}$ were reseeded in tissue culture plate for 3 days. (D) hMSCs plated in ultra-low-adherence tissue culture plate for $24 \mathrm{~h}$. (E) hMSCs plated in ultra-low-adherence tissue culture plate for $72 \mathrm{~h}$. (F) Nonadherent culture cells from panel E at $72 \mathrm{~h}$ were reseeded in the tissue culture plates for 3 days. Original magnification, x10. hMSC, human mesenchymal stem cells; Ad, adherent culture; Nonad, nonadherent culture.

properties of the hMSCs isolated in the present study, their clonogenic and multipotent lineage potential were evaluated by CFU-F and in vitro differentiation assays respectively. CFUs were detected within the cell population, and cells within individual colonies exhibited fibroblast-like morphology (Fig. 1A), indicating good self-renewing properties of the isolated cell populations. The differentiation assays of passage- 2 hMSCs demonstrated that the cells were able to generate adipocytes (Fig. 1B), osteoblasts (Fig. 1C), and chondrocytes (Fig. 1D) in vitro. These findings suggest that the cells exhibit the multipotent differentiation potential characteristic of MSCs. In addition, adherent and nonadherent-cultured cells following $72 \mathrm{~h}$ culture also demonstrated successful adipocyte (Fig. 1E) and chondrocyte (Fig. 1F) differentiation.
Flow cytometry analysis of MSC surface markers. The expression of surface markers, including CD11b, CD14, CD34, CD45, CD73, CD90 and CD105, has been previously reported as a means to confirm the correct cell identity for MSCs (13). Therefore, expression of these surface markers was analyzed by flow cytometry in passage- 2 cells. Greater than 99\% of passage- 2 cells expressed CD73, CD90, and CD105 (Fig. 2A-C). By contrast, less than $1 \%$ of cells expressed CD11b, CD14, CD34 and CD45 (Fig. 2D-G, respectively). These findings suggest that the cells isolated in the present study are enriched in phenotypically defined hMSCs.

Morphology of adherent-cultured and nonadherent-cultured hMSCs. hMSCs seeded in tissue culture plates (at 
A
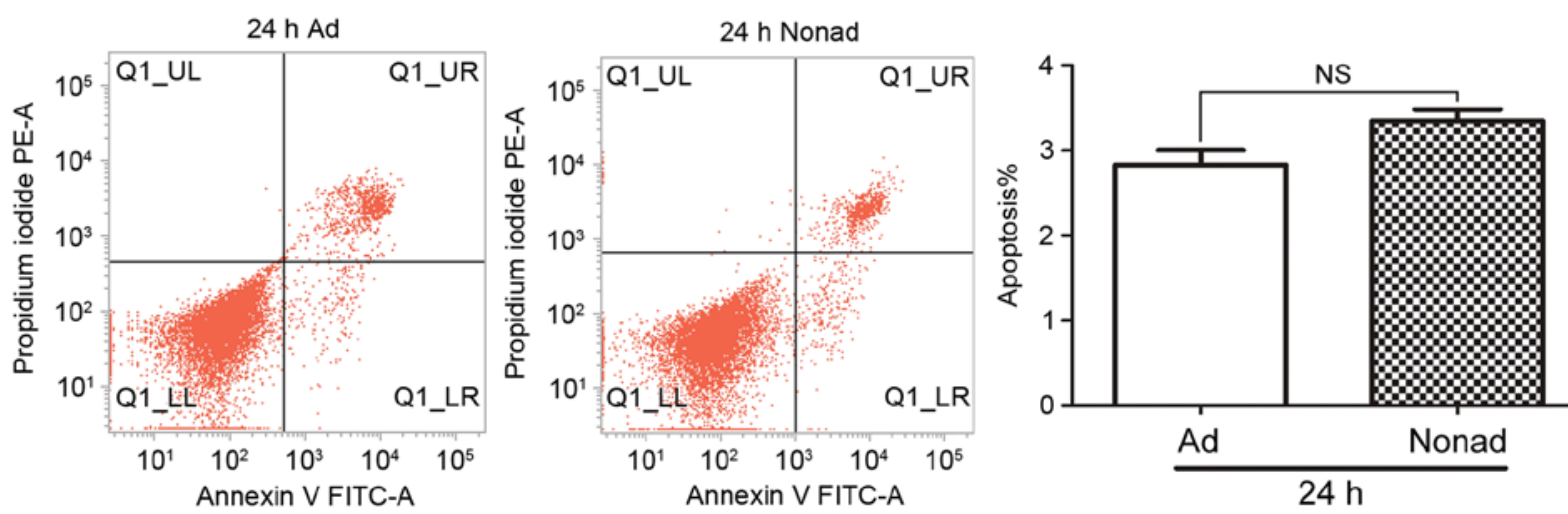

B
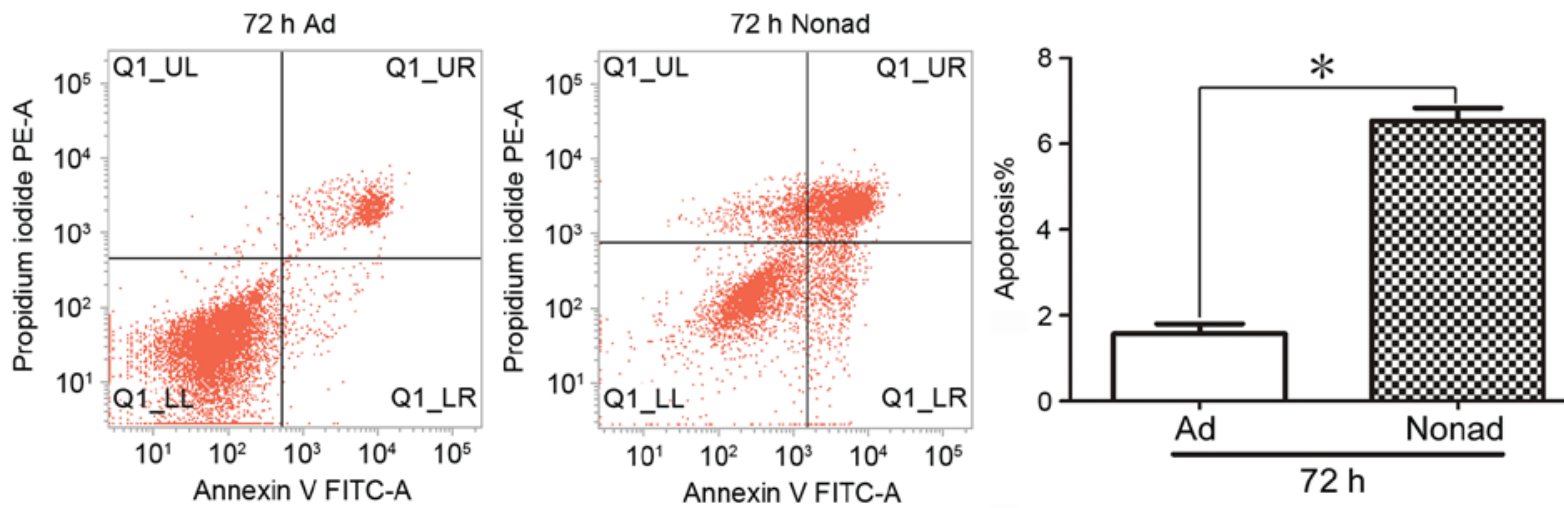

Figure 4. Effect of nonadherent culture on apoptosis in hMSCs. Adherent and nonadherent-cultured hMSCs at 24 or 72 h were stained with Annexin V-FITC/PE followed by flow cytometric analysis. (A) Representative plots and quantification of \% apoptotic cells in the total cell population in adherent and nonadherent-cultured hMSCs at $24 \mathrm{~h}$. (B) Representative plots and quantification of \% apoptotic cells in the total cell population in adherent and nonadherent-cultured hMSCs at $72 \mathrm{~h}$. Results are presented from three independent experiments. * $\mathrm{P}<0.05$ compared with adherent-cultured cells. hMSC, human mesenchymal stem cells; FITC, fluorescein isothiocyanate; PE, phycoerythrin; Ad, adherent culture; Nonad, nonadherent culture; NS, not significant.

2,500 cells/well) were attached to the bottom of the plates and exhibited flat morphology following 24 (Fig. 3A) and $72 \mathrm{~h}$ (Fig. 3B) of culture. However, hMSCs seeded in the ultra-low-adherence culture plates appeared suspended and scattered throughout the medium, and exhibited a rounded morphology, following 24 (Fig. 3D) and 72 h (Fig. 3E) of culture. In order to test whether this morphology change was a transient or permanent feature, adherent and nonadherent cells were harvested at $72 \mathrm{~h}$ and then reseeded in tissue culture plates. Following incubation for 3 days in adherent culture conditions, hMSCs reseeded from the nonadherent cultures exhibited a similar flat morphology (Fig. 3F) to the ones reseeded from the adherent cultures (Fig. 3C), suggesting that nonadherent cells were capable of adherent growth and fibroblast-like morphology.

Effect of nonadherent culture conditions on apoptosis in hMSCs. Apoptosis of passage- 3 hMSCs was analyzed by flow cytometry in three independent repeats. At $24 \mathrm{~h}$, the \% of apoptotic cells in nonadherent-cultured cells was not significantly different compared with the control adherent-cultured cells (P>0.05; Fig. 4A). At $72 \mathrm{~h}$, the \% of apoptotic cells was significantly higher in the nonadherent-cultured cells compared with the adherent-cultured cells $(\mathrm{P}<0.05$; Fig. 4B), indicating that nonadherent culture conditions result in increased apoptosis in hMSCs.
Effect of nonadherent culture conditions on caspase mRNA expression in hMSCs. RT-qPCR was used to compare levels of caspase-3, -7, -8, and-9 mRNA expression in adherent and nonadherent-cultured cells at 24 and $72 \mathrm{~h}$. At $24 \mathrm{~h}$, mRNA expression levels of all the caspases examined were not significantly different in nonadherent-cultured cells compared with the adherent-cultured cells ( $\mathrm{P}>0.05$; Fig. 5A-D, respectively). At $72 \mathrm{~h}$ however, mRNA expression levels of caspase-3, -7, and -9 were significantly increased in nonadherent-cultured cells compared with the control adherent-cultured cells $(\mathrm{P}<0.05$; Fig. 5A, B and D, respectively). Expression of caspase-8 did not differ significantly between the two groups at either time point (P>0.05; Fig. 5C).

Effect of nonadherent culture conditions on expression of caspase proteins in hMSCs. Since caspase-3, -7, and -9 mRNA expression at $72 \mathrm{~h}$ differed between adherent and nonadherent-cultured cells, protein levels were also examined by western blot analysis. The results demonstrated that at $24 \mathrm{~h}$, the protein expression levels of caspases 3, 7, 8, and 9 did not differ significantly between culture conditions ( $\mathrm{P}>0.05$; Fig. 6A). At $72 \mathrm{~h}$, levels of caspase-3, caspase-7, and caspase- 9 proteins were significantly lower in the nonadherent-cultured cells compared with the adherent-cultured cells $(\mathrm{P}<0.05$; Fig. $6 \mathrm{~B})$, while the level of caspase- 8 protein did not differ significantly between the two populations at 24 or $72 \mathrm{~h}$ ( $\mathrm{P}>0.05$; Fig. 6). 

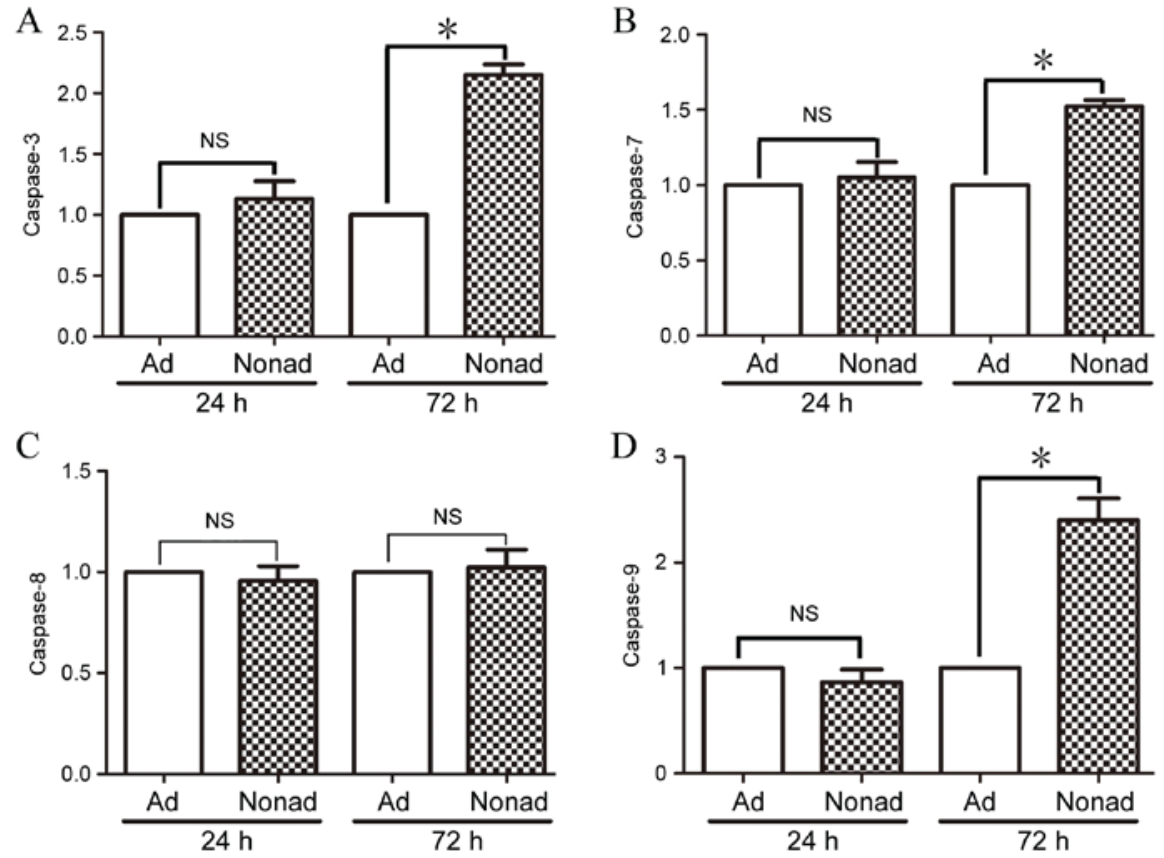

Figure 5. Effect of nonadherent culture on mRNA expression of caspases. mRNA expression levels of (A) caspase-3, (B) caspase-7, (C) caspase-8 and (D) caspase-9 genes were evaluated in adherent-cultured and nonadherent-cultured hMSCs at 24 and $72 \mathrm{~h}$ by reverse transcription-quantitative polymerase chain reaction. Data were averaged from three independent experiments. " $\mathrm{P}<0.05$, with comparisons indicated by brackets. hMSC, human mesenchymal stem cells; Ad, adherent culture; Nonad, nonadherent culture; NS, not significant.

$\mathbf{A}$
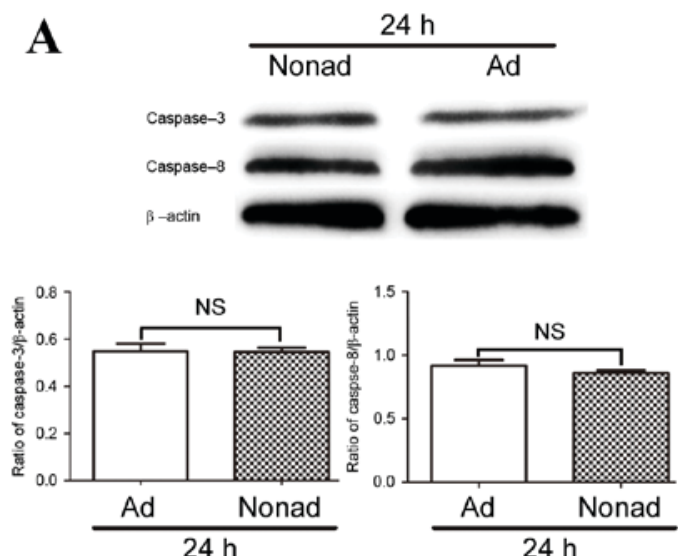

B
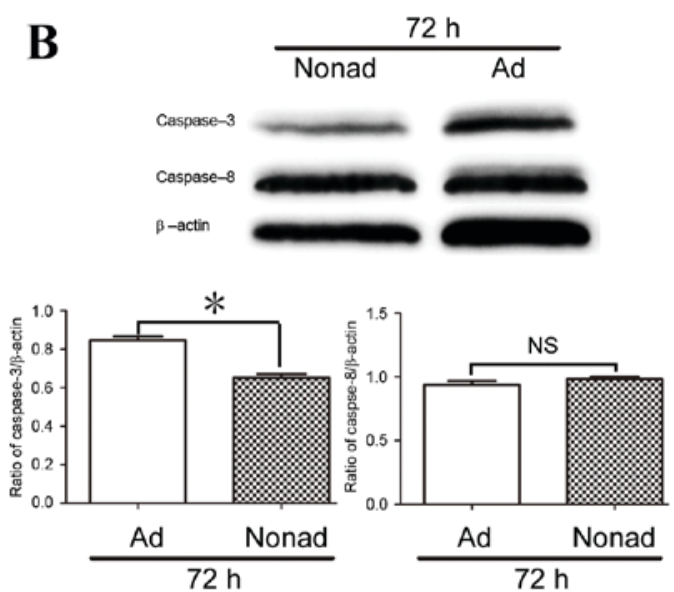
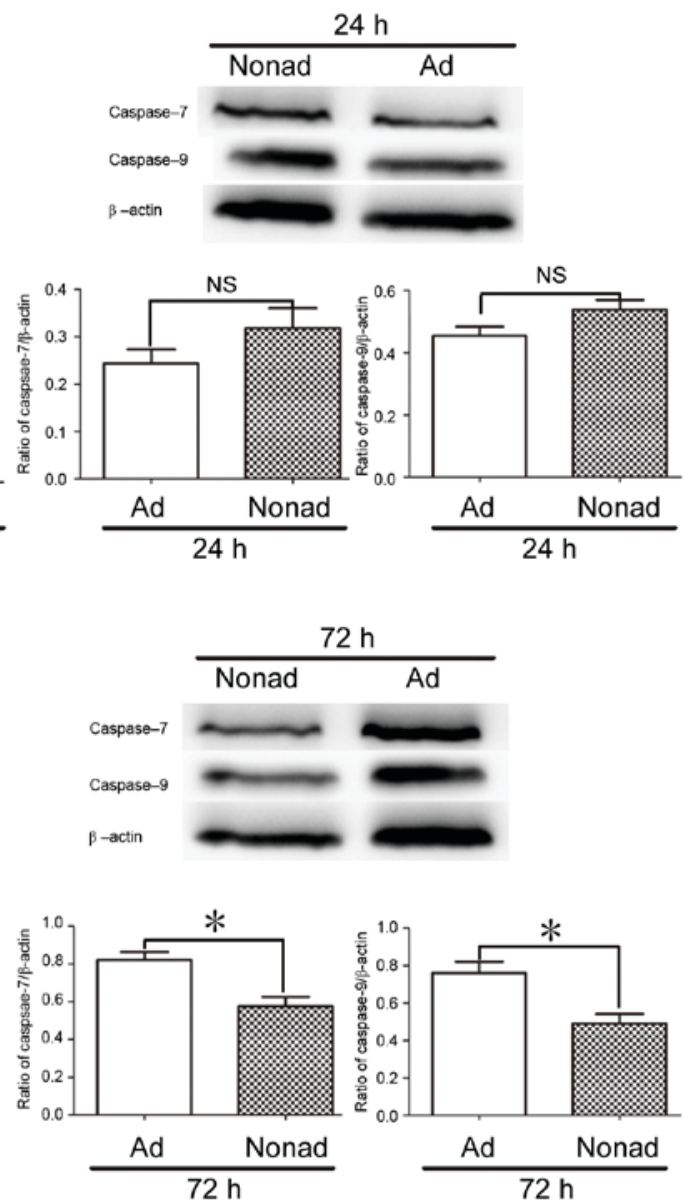

Figure 6. Effect of nonadherent culture on protein expression of caspases. Western blot analysis of protein expression levels of caspase-3, -7 , -8 and- 9 in adherent and nonadherent-cultured hMSCs at (A) 24 and (B) $72 \mathrm{~h}$ of culture. $\beta$-actin was used as a loading control, and expression levels of the target proteins were determined relative to the levels of $\beta$-actin. Data were averaged from three independent experiments. * $\mathrm{P}<0.05$ compared with adherent-cultured cells hMSC, human mesenchymal stem cells; Ad, adherent culture; Nonad, nonadherent culture; NS, not significant. 


\section{Discussion}

Numerous studies have demonstrated the efficiency of MSCs in stem cell therapy recovery of the infarcted myocardium, however, a major limitation of this method is that MSCs are short-lived following transplantation $(5,14)$. Apoptosis of MSCs following transplantation is an important factor in this lack of long-term survival. In the present study, a previously described method was utilized by seeding MSCs in ultra-low-adherence culture plates to assess the apoptotic properties of hMSCs removed from adherent culture conditions.

To isolate hMSCs, bone marrow was aspirated from the posterior iliac crest. The criteria used to define hMSCs were as follows: i) MSCs must be adherent when maintained under standard culture conditions, ii) MSCs must express CD105, CD73, and CD90, and lack expression of CD45, CD34, CD14 and CD11b surface molecules, and iii) MSCs must be able to differentiate to osteoblasts, adipocytes and chondroblasts in vitro (13). The isolated cells were confirmed for surface marker expression by flow cytometric analysis and for clonogenicity and multipotency by in vitro $\mathrm{CFU}-\mathrm{F}$ and differentiation assays. These findings demonstrated that the isolated cells had the characteristics of hMSCs. In addition, adherent and nonadherent-cultured cells at $72 \mathrm{~h}$ also demonstrated adipocyte and chondrocyte differentiation potential and plastic adherence ability.

Apoptosis rates were then compared between nonadherent and adherent-cultured cells at 24 and $72 \mathrm{~h}$ using flow cytometric analysis. The $\%$ of apoptotic cells in nonadherent-cultured cells increased significantly compared with adherent-cultured cells at $72 \mathrm{~h}$. These data indicated that apoptosis increased in hMSCs following removal from adherent culture conditions. Baksh et al $(15,16)$ demonstrated that bone marrow-derived nonhematopoietic progenitor cells proliferate in a cytokine-dependent manner, as individual cells in stirred suspension cultures. In the present study, neither adherent or nonadherent cultures contained any exogenously added cytokines, and both were cultured in static conditions. In addition, hMSCs were isolated by adherence to plastic, the hMSCs were a different cell population compared with nonhematopoietic progenitor cells. This implies that some cytokines may be critical for the survival of MSCs following removal from adherent culture conditions.

Mammalian cell apoptosis is primarily activated via two distinct pathways, the intrinsic and extrinsic apoptotic pathways (17). Caspase- 8 and -3 are involved in the extrinsic apoptotic pathway (18), while caspase-3, -7 and -9 participate in the intrinsic apoptotic pathway (19). In the present study, the protein levels of caspase-3, -7 , and -9 were significantly reduced following $72 \mathrm{~h}$ of nonadherent culture, whereas caspase- 8 was unchanged. These data indicate that the intrinsic apoptotic pathway may be involved in the apoptosis of nonadherent hMSCs. Caspase- 9 is activated by cleavage, then acts on downstream targets, including caspase- 3 and $-7(19,20)$. The levels of mRNA expression for caspase-3, -7, and -9 genes were increased at $72 \mathrm{~h}$. By contrast, due to protein cleavage, the protein expression levels of caspase-3, -7, and -9 were decreased in nonadherent-cultured cells compared with control adherent-cultured cells. Taken together, these data suggest that caspase-3, -7 and -9 proteins may be involved in regulating apoptosis in hMSCs following removal from plastic-adherent culture conditions, through the intrinsic apoptotic pathway.

Previous studies indicated that ischemia, inflammatory response, hypoxia and oxidative stress are the major factors impairing MSC survival following transplantation $(6,7,21)$. The present study demonstrated another possible reason for the low survival of MSCs. In conclusion, loss of adherence in vitro increases apoptosis in hMSCs, through activation of caspase-3, -7 and -9 . The results of the present study may provide novel insights into factors affecting MSC survival following transplantation.

\section{Acknowledgements}

We thank Hong Qian of the Center for Hematology and Regenerative Medicine of Karolinska Institute (Stockholm, Sweden) for valuable advice in this study. This study was supported by grants from the International Program of Project 985, Sun Yat-sen University and the 'One Hundred Talented Scholars' of Sun Yat-sen University (grant no. F002009011) and Zhongshan Postdoctoral Sustentation Fund (grant no. E2015681).

\section{References}

1. Satija NK, Singh VK, Verma YK, Gupta P, Sharma S, Afrin F, Sharma M, Sharma P, Tripathi RP and Gurudutta GU: Mesenchymal stem cell-based therapy: A new paradigm in regenerative medicine. J Cell Mol Med 13: 4385-4402, 2009.

2. Novotny NM, Ray R, Markel TA, Crisostomo PR, Wang M, Wang Y and Meldrum DR: Stem cell therapy in myocardial repair and remodeling. J Am Coll Surg 207: 423-434, 2008.

3. Deuse T, Peter C, Fedak PW, Doyle T, Reichenspurner H, Zimmermann WH, Eschenhagen T, Stein W, Wu JC, Robbins RC and Schrepfer S: Hepatocyte growth factor or vascular endothelial growth factor gene transfer maximizes mesenchymal stem cell-based myocardial salvage after acute myocardial infarction. Circulation 120 (11 Suppl): S247-S254, 2009.

4. Rabani V, Shahsavani M, Gharavi M, Piryaei A, Azhdari Z and Baharvand $\mathrm{H}$ : Mesenchymal stem cell infusion therapy in a carbon tetrachloride-induced liver fibrosis model affects matrix metalloproteinase expression. Cell Biol Int 34: 601-605, 2010.

5. Burlacu A: Tracking the mesenchymal stem cell fate after transplantation into the infarcted myocardium. Curr Stem Cell Res Ther 8: 284-291, 2013.

6. Wei H, Li Z, Hu S, Chen X and Cong X: Apoptosis of mesenchymal stem cells induced by hydrogen peroxide concerns both endoplasmic reticulum stress and mitochondrial death pathway through regulation of caspases, p38 and JNK. J Cell Biochem 111: 967-978, 2010

7. Potier E, Ferreira E, Meunier A, Sedel L, Logeart-Avramoglou D and Petite H: Prolonged hypoxia concomitant with serum deprivation induces massive human mesenchymal stem cell death. Tissue Eng 13: 1325-1331, 2007.

8. Vunjak-Novakovic G and Scadden DT: Biomimetic platforms for human stem cell research. Cell Stem Cell 8: 252-261, 2011.

9. Deng B, Deng W, Xiao P, Zeng K, Zhang S, Zhang H, Deng DY and Yang Y: Nonadherent culture method downregulates stem cell antigen-1 expression in mouse bone marrow mesenchymal stem cells. Exp Ther Med 10: 31-36, 2015.

10. Qian H, Le Blanc K and Sigvardsson M: Primary mesenchymal stem and progenitor cells from bone marrow lack expression of CD44 protein. J Biol Chem 287: 25795-25807, 2012.

11. Livak KJ and Schmittgen TD: Analysis of relative gene expression data using real-time quantitative PCR and the 2(-Delta Delta C(T)) method. Methods 25: 402-408, 2001.

12. Schneider CA, Rasband WS and Eliceiri KW: NIH image to imageJ: 25 years of image analysis. Nat Methods 9: 671-675, 2012 . 
13. Dominici M, Le Blanc K, Mueller I, Slaper-Cortenbach I, Marini F, Krause D, Deans R, Keating A, Prockop Dj and Horwitz E: Minimal criteria for defining multipotent mesenchymal stromal cells. The International society for cellular therapy position statement. Cytotherapy 8: 315-317, 2006.

14. Sheikh AY, Huber BC, Narsinh KH, Spin JM, van der Bogt K, de Almeida PE, Ransohoff KJ, Kraft DL, Fajardo G, Ardigo D, et al: In vivo functional and transcriptional profiling of bone marrow stem cells after transplantation into ischemic myocardium. Arterioscler Thromb Vasc Biol 32: 92-102, 2012.

15. Baksh D, Davies JE and Zandstra PW: Adult human bone marrow-derived mesenchymal progenitor cells are capable of adhesion-independent survival and expansion. Exp Hematol 31: 723-732, 2003

16. Baksh D, Zandstra PW and Davies JE: A non-contact suspension culture approach to the culture of osteogenic cells derived from a CD49elow subpopulation of human bone marrow-derived cells. Biotechnol Bioeng 98: 1195-1208, 2007.

17. Parrish AB, Freel CD and Kornbluth S: Cellular mechanisms controlling caspase activation and function. Cold Spring Harb Perspect Biol 5: pii: a008672, 2013.
18. Vanden Berghe T, van Loo G, Saelens X, Van Gurp M, Brouckaert G, Kalai M, Declercq W and Vandenabeele P: Differential signaling to apoptotic and necrotic cell death by Fas-associated death domain protein FADD. J Biol Chem 279: 7925-7933, 2004.

19. Rowinsky EK: Targeted induction of apoptosis in cancer management: The emerging role of tumor necrosis factor-related apoptosis-inducing ligand receptor activating agents. J Clin Oncol 23: 9394-9407, 2005.

20. Philchenkov A, Zavelevich M, Kroczak TJ and Los M: Caspases and cancer: Mechanisms of inactivation and new treatment modalities. Exp Oncol 26: 82-97, 2004.

21. Deschepper M, Oudina K, David B, Myrtil V, Collet C, Bensidhoum M, Logeart-Avramoglou D and Petite H: Survival and function of mesenchymal stem cells (MSCs) depend on glucose to overcome exposure to long-term, severe and continuous hypoxia. J Cell Mol Med 15: 1505-1514, 2011. 